\title{
Intrapulse analysis of radar signal
}

\author{
A. Pieniężny \& S. Konatowski \\ Department of Electronics, Military University of Technology, Poland
}

\begin{abstract}
ELINT/ESM type of electronic intelligence in the primary layer uses parameters measurements of intercepted radar signals. Nowadays modern radar uses more and more complex waveforms. Some waveforms are developed intentionally to make their intercept almost impossible. The main distinctive features of modern radar signal are hidden in its time-frequency structure. In the near past the problem of radar signal feature extraction was considered in time or frequency domain separately, because radar waveforms were relatively simple. Today, however, the signals should be observed simultaneously in both domains. Timefrequency distribution concept offers a new approach in radar signals classification/identification. The paper presents some results of compressive concept and Hough transform application to intra-pulse modulation analysis of radar signals. Linear frequency modulation within the pulse was considered.
\end{abstract}

Keywords: signal spectrum, chirp transform, compressive receiver, Hough transform, intra-pulse modulation.

\section{Introduction}

Electronic intelligence (ELINT) and electronic warfare support measures (ESM) devices for modern electronic warfare (EW) systems detect the presence of potentially hostile radar (e.g. [13, 14]). The first class of EW systems (ELINT) performs mainly the identification and location tasks of unknown emitters with purpose to determine their parameters that are subsequently included to the threat library. ELINT/ESM systems operation and effectiveness is not only determined by their technical performance quality but also by threat libraries correctness. ESM system itself is particularly very sensitive to this factor due to automatic mode of operation. Modern battlefield requirements are very demanding with respect to ELINT/ESM performance and reliability. This type of equipment has to be highly sophisticated with respect to their hardware and software 
architecture. The state of the art in microwave receiver's technology and in signal processing methods and techniques gradually meet modern battlefield challenges. The typical ELINT/ESM equipment structure usually consists of antenna (antennas), microwave receiver typically providing two intermediate frequency (IF) outputs and demodulating section processing these IF outputs. Receiver is used for radar signals interception whereas the demodulating section is used to determine signal parameters. Intrapulse modulation is becoming distinctive feature of a signal. Considering this parameter it seems reasonable to introduce it as an important factor of deinterleaving process. Deinterleaving process basically is based on signal time parameters measurement. The following are time parameters considered as a primary parameters (measured for each pulse): (TOA)-time of arrival, (PW)-pulse width, (A)-amplitude, (RF)-radiofrequency (carrier frequency), (FMOP)-frequency modulation on pulse. Time parameters are associated with (AOA)-angle of arrival. For each pulse the specific description, $\mathrm{PDW}$-pulse descriptor word, containing primary parameters is created. On the base of PDW the process of deinterleaving is performed. Today to provide low probability of interception and identification of radar signal, radar waveforms are more and more complex. The waveform complexity is realized mainly by intrapulse modulation. Thus the signal frequency structure has become a very important signal descriptor. Time-frequency distribution concept offers a new approach in radar signals processing. Wideband signals compression principle using surface acoustic (SAW) dispersive delay lines (DDL), to measure instantaneous spectrum of short pulsed radar signals is presented. Furthermore the results of image processing techniques, represented by Hough transform, with application to intra-pulse modulation analysis of radar signals have been shown. Linear frequency modulation within the pulse was considered

\section{Compressive receiver concept}

A good understanding of the compressive receiver (CR) performance requires a short discussion of the fundamentals of chirp transform. The chirp transform is based on classical Fourier transform in which linear dependency between frequency and time (delay) has been introduced $[1,2,4,8]$

$$
F(\omega)=\int_{-\infty}^{+\infty} f(t) e^{-j \omega t} d t . \text { and } \omega=2 \mu \tau
$$

where: $f(t)$ - input signal, $\omega$ - frequency due to time $\tau, \mu$ - frequency-to-time conversion factor. After substitution the equality $\omega=2 \mu \tau$, and applying the identity $2 \tau t=\tau^{2}+t^{2}-(\tau-t)^{2}$, and suitable factorization, the first equation is converted in a frequency-to-time scaling problem

$$
S(\tau)=\left\{\int_{-\infty}^{+\infty} s(t) e^{-j \mu t^{2}} e^{-j \mu(\tau-t)^{2}} d t\right\} e^{-j \mu \tau^{2}}
$$

where with comparison to the equation (1), $F(\omega)$ and $f(t)$ have been substituted by $S(\tau)$ and $s(t)$ respectively. The last expression describes the full chirp 
algorithm. It has electrical implementation, presented in Fig.1, which requires three operations. First, the input signal $s(t)$ is multiplied by a chirp reference signal. Secondly, the resultant product is convolved in pulse compression filter with an equal but opposite frequency-to-time slope value $\mu$. Thirdly, a multiplication by a second chirp, which removes the phase distortion introduced by the first chirp. In spectral analysis application, such as a signal spectrum measurement, the second chirp multiplication is omitted since the desired information is the envelope of the Fourier transform. In such a case the chirp algorithm (transform) becomes the version which produce signal power spectrum only

$$
V(\tau)=\int_{-\infty}^{+\infty} s(t) e^{-j \mu t^{2}} e^{-j \mu(\tau-t)^{2}} d t
$$

The chirp algorithm arrangement (3), referred to us as M-C (multiplyconvolve) release has practical hardware implementation including chirp generator, mixer, compression filter, amplifier and envelope detector. The radio frequency (RF) signal is multiplied by the linear sweeping reference signal to produce of the linear sweeping intermediate frequency. This signal is subsequently applied to the pulse compression filter connected with the intermediate frequency (IF) amplifier. The compressed output signal envelope is reproduced by the detection circuit. Output video signal appears at time determined by the input signals frequency values. It reflects instantaneous spectrum of input signal. The $\mathrm{M}-\mathrm{C}$ algorithm, is recommendable for the analysis of short pulsed radar signals. To clarify the CR performance some registrations has been made. The results are presented in Fig. 2 to Fig 6. Fig. 4 and Fig. 5 show one of the most significant feature of the $\mathrm{CR}$, which is ability to analyze signals that are simultaneously present at its input. Fig.3 presents CR output video signals for pulsed wideband linear frequency modulated (LFM) signals and Fig.6 shows CR response for single pulse of intercepted narrowband signal.
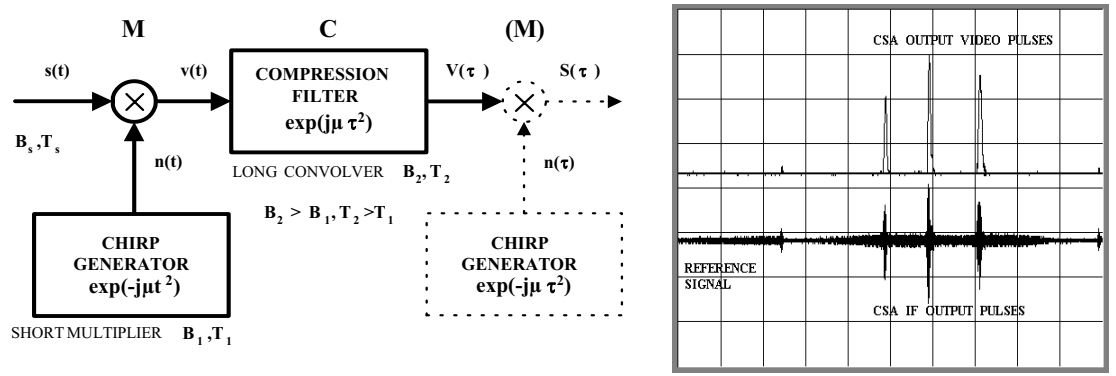

Figure 1: Chirp algorithm arrangement.

Figure 2:

CR performance for CW signals, $(2 \mu \mathrm{s} / \mathrm{div})$. 


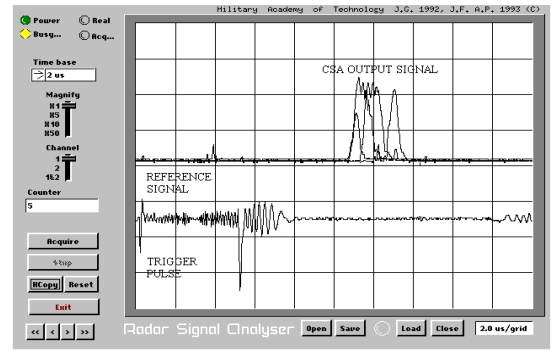

Figure 3: $\quad \mathrm{CR}$ performance for pulsed LFM signal, $(2 \mu \mathrm{s} / \mathrm{div})$.

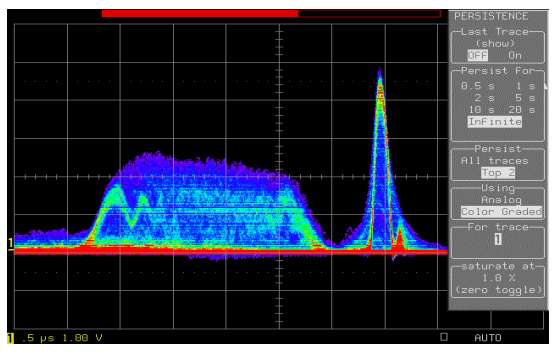

Figure 5: $\quad$ CR output signals for two input signals: pulsed LFM signal and CW signal, $0,5 \mu \mathrm{s} / \mathrm{div}$.

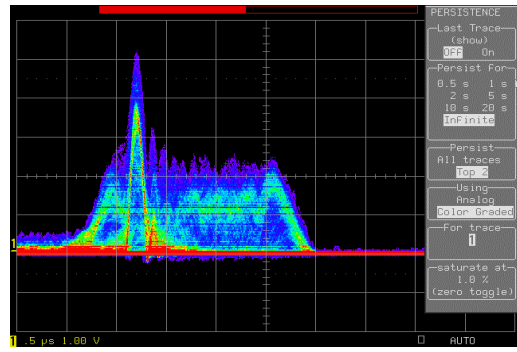

Figure 4: $\quad$ CR output signals for two input signals: pulsed LFM signal and $\mathrm{CW}$ signal, $0,5 \mu \mathrm{s} / \mathrm{div}$.

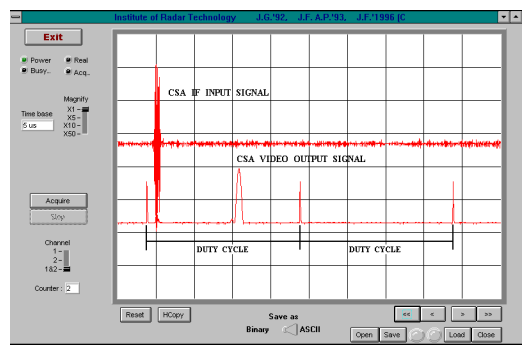

Figure 6: $\quad \mathrm{CR}$ input and output signals - pulsed unmodulated signal, $5 \mu \mathrm{s} / \mathrm{div}$.

The CR output signal reflects instantaneous spectrum of the analysed signal. It performs signal's analysis in particular time samples refer to us as duty cycles. This results signal spectrum averaging over reference signal time and bandwidth. In other words, the $\mathrm{CR}$ output signal expresses signal energy being under analysis. Thus, with respect to the time-frequency distribution terms, to collect a total signal energy it is necessary to gather signals existing in several duty cycles of the CR. Therefore the time-frequency signal representation will be referred to the plane (duty cycle number, frequency expressed by time) over which the CR output signal will be evaluated. As resulted from the registrations, the $\mathrm{CR}$ operation consists of several duty cycles in which instantaneous spectrum of a signal is yielded. For particular cycle, signal spectrum is represented by video pulse position relatively to the beginning of a reference signal. Pulse position is determined by the input signal frequency value. Thus to evaluate spectrum it is sufficient to measure the time intervals between CR output video pulse and the 
pulse triggering the reference signal. Another factor that should be taken under attention is CR output pulse amplitude variability.

\section{Compressive receiver output signal processing}

The CR output is followed by many short pulses with positions determined by the input signal frequency value. There exist also side lobes associated with these pulses. Thus to measure the frequency of the input signal the measuring of the main pulse centre with simultaneous neglecting of side lobes is required. To determine the signal frequency, the time parameters measurements should be performed. The simplest approach is to compare the CR output signal with fixed thresholds. When the processed pulse breaks these thresholds, it will be declared a legible output. This approach includes two main shortcomings. Firstly, the amplitude of the output pulse changes with the input signal level. Secondly the fixed thresholds detection scheme does not distinguish between main and side lobes in general. Thus to overcome problems of the CR output signal processing digitizing technique is recommended. To proceed examinations the CR practical model has been used [3, 5-7].

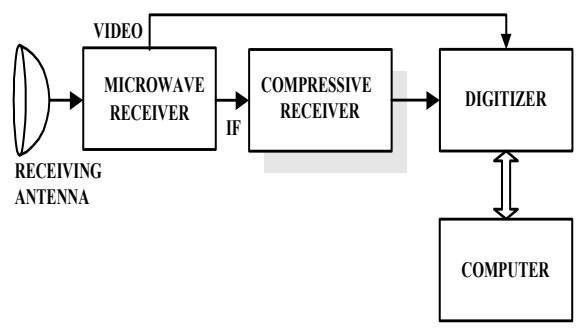

Figure 7: Measurement system lock diagram.

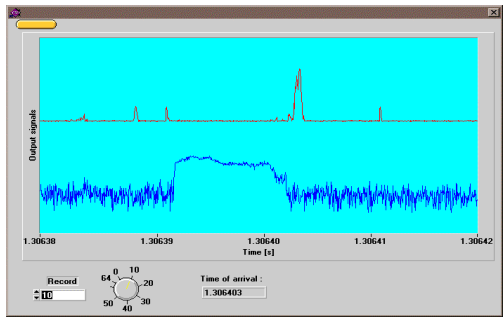

Figure 8: $\quad \mathrm{CR}$ and receiver video outputs, (pulsed LFM signal, pulse width $10 \mu \mathrm{s})$.

The measurement system block diagram is presented in Fig.7. Some results of the examinations are presented in Fig.9-12 (in Fig.10 upper trace represents CR output, lower trace represent receiver video output). Fig.8 combines instantaneous video of the intercepted signal and its instantaneous spectrum represented by the CR output video for the single pulse. Fig.9-12 present spectrum two types of radar signals - pulsed linear frequency modulated and narrowband (unmodulated) (in Fig.10, 12 record number denotes time, sample number denotes frequency). Number of pulses under analysis depends on digitizer threshold. As it is seen in the case of pulse train it is possible to estimate frequency deviation on pulse (on the base of pulse train), however it is not possible to determine modulation type. 


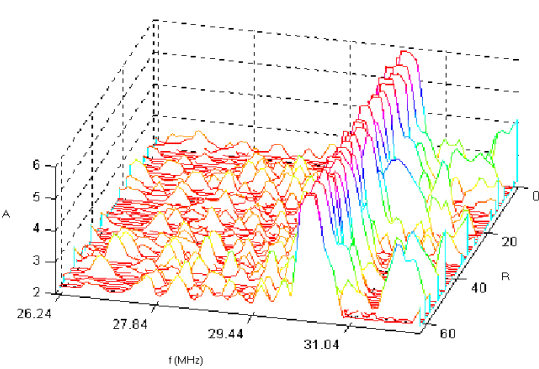

Figure 9: $\quad$ CR output signals, pulsed unmodulated signal (pulse width $5 \mu \mathrm{s})$.

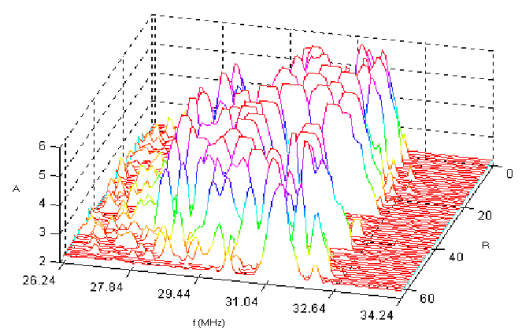

Figure 11: CR output signals, pulsed LFM signal

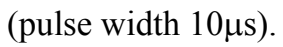

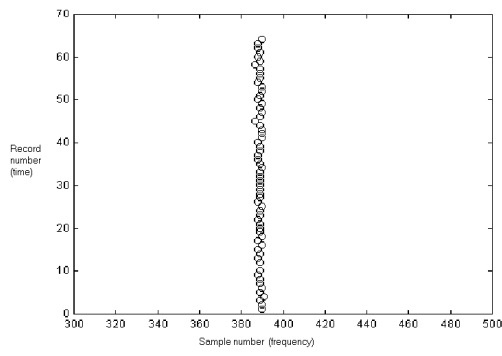

Figure 10: CR output signals pulsed unmodulated signal.

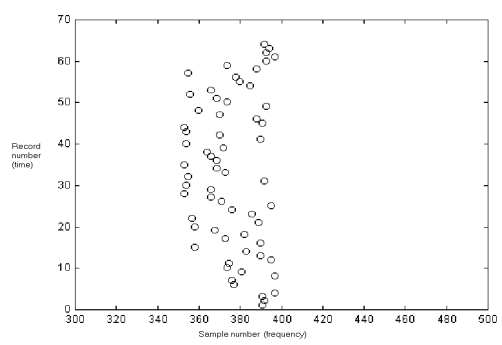

Figure 12: CR output signals, pulsed LFM signal (pulse width $10 \mu \mathrm{s}$ ).

\section{Wigner-Hough transform}

Methods of time-frequency analysis enable to create radar signal image representing its instantaneous frequency $[11,12]$. Processing such an image one can determine intrapulse parameters of radar signal. Hough transform is a technique used in image processing, that could also be implemented here [15]. This technique enables to analyze global features of an image of the base of local ones (point is the favourite one). The method relay on lines detection that could be represented in parametric form as straight lines circles, polynomials [9, 10, 15]. The problem analyzed in the paper may be considered as a separate application of two stages: firstly the Wigner-Ville transform $(W V)$ is calculated $[11,12]$ and secondly the Hough transform is used. It is possible to apply here joint Wigner-Hough transform. Such method relays on Hough transform application to Wigner-Ville transformation of a signal. Particularly the method will be used to analyze multi-component signals with linear intra-pulse frequency modulation. The aim of the paper is to present WHT performance with application to multi-component radar signals processing. Wigner-Hough 
transform of complex signal $s(t)$ is defined as a transformation from time domain to parameters $(f, g)$ domain accordingly to the expression [10]:

$$
W H_{s}(f, g)=\int_{-\infty}^{\infty} \int_{-\infty}^{\infty} s(t+\tau / 2) s^{*}(t-\tau / 2) \cdot e^{-j 2 \pi(f+g t) \tau} d \tau d t
$$

The transform defined by the equation (4) one can consider as integral of Wigner-Ville transform:

$$
W H_{s}(f, g)=\int_{-\infty}^{\infty} \int_{-\infty}^{\infty} W_{s}(t, v) \delta(v-f-g t) d t d v=\int_{-\infty}^{\infty} W_{s}(t, f+g t) d t
$$

where $\delta(v-f-g t)$ is delta function and Wigner-Ville transform is defined accordingly [11]:

$$
W_{s}(t, f)=\int_{-\infty}^{\infty} s(t+\tau / 2) s^{*}(t-\tau / 2) e^{-j 2 \pi f \tau} d \tau
$$

For practical applications discrete Wigner-Hough transform is needed. The discrete $W H T$ for sequence $x(n), n=0,1, \ldots, N-1$ (where $N$ is even) is given by the equation [10]:

$$
\begin{aligned}
& W H_{x}(f, g)=\sum_{n=0}^{N / 2-1} \sum_{k=-n}^{n} x(n+k) x^{*}(n-k) e^{-j 4 \pi k(f+g n)}+ \\
& +\sum_{n=N / 2}^{N-1} \sum_{k=-(N-1-n)}^{N-1-n} x(n+k) x^{*}(n-k) e^{-j 4 \pi k(f+g n)}
\end{aligned}
$$

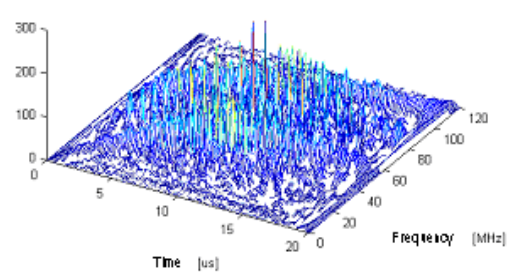

Figure 13: WV of two LFM signals embedded in noise.

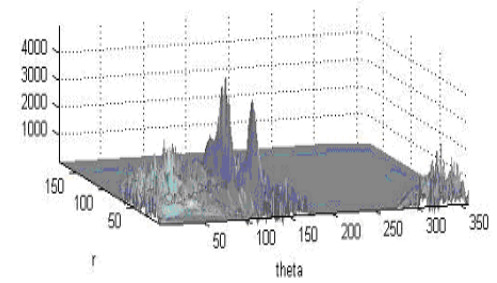

Figure 14: WHT of two LFM signals.

As it results from (7), WHT for signals with linear frequency modulation $(L F M)$ reaches the maximum value in the points of coordinates $\left(f_{0}, g_{0}\right)$. It denotes that detection and parameter estimation of $L F M$ signals embedded in noise one can consider as a peak search in parameter domain $(f, g)$. An example of WHT application in the case of multi-component signals interfered by additive Gaussian noise is presented on Fig.13, 14. Fig.13 presents $W V$ of two LFM signals for signal-to-noise ratio $(S N R)$ equal zero $\mathrm{dB}$. Both signals are $L F M$ signals and have the same amplitude, the same frequency-to-time slope but different frequency carriers. Signals were simulated, however they could reflect possible real values. On the base of $W V$ image it is difficult to identify the signal type. When $W H T$ is used then in transformed $W V$ image two peaks, representing analyzed signals, are observed. Fig.14 shows two important advantages of WHT: 
first - in parameters domain peaks of signals are observed because cross terms were canceled, second - WHT gives integration gain with relation to noise as a result of integration.

Parameters estimation of multi-component LFM signals is performed simultaneously for all elements in $(f, g)$ plane represented here by $r$ and theta (Fig.14). When peak exceeds predetermined threshold, it is declared that LFM signal is present and its parameters $f_{0}$ and $g_{0}(r$,theta $)$ are coordinates of the peak.

\section{Noise influence on WHT transform}

When signal is embedded in noise the WHT is becoming random variable $W H T S+v\left(f_{0}, g_{0}\right)$. The peak of WHT moves to the point of coordinates $\left(f_{0}+\delta_{f}\right.$, $g_{0}+\delta_{g}$ ). In this case the $W H T$ properties are described by output $S N R$ defined accordingly to $[10]$ :

$$
S N R_{\text {OUT }}=\frac{\left|W H T_{s}\left(f_{0}, g_{0}\right)\right|^{2}}{\operatorname{var}\left\{W H T_{s+v}\left(f_{0}, g_{0}\right)\right\}}
$$

As it results from expression (8) output $S N R$ is defined by $\operatorname{WHTs}(f, g),(W H T$ of signal) and by $W H T s+v(f, g)$, (WHT of signal embedded in noise). Signal parameters accuracy estimation is determined by variance of random variables $\delta_{f}$ and $\delta g$. Assuming that noise is zero mean, white Gaussian process the following expression for $S N R_{O U T}$ is received [10]:

$$
S N R_{\text {OUT }}=\frac{\frac{N^{4} A^{4}}{4}}{\frac{N^{3} A^{2} \sigma_{n}^{2}}{2}+\frac{N^{2} \sigma_{n}^{4}}{2}}=\frac{\frac{N^{2}}{2} S N R_{I N}^{2}}{N \cdot S N R_{I N}+1}
$$

where input $S N R_{I N}$ is defined as $A^{2} / \sigma^{2}{ }_{n}, A-$ signal amplitude, $\sigma_{n}^{2}$ - noise variance, $N$ - number of samples. Last expression reveals the threshold effect. When input $S N R$ is high $\left(S N R_{I N}>>1\right)$, the expression (5) is approximated by $S N R_{O U T}=N S N R_{I N} / 2$. The integration gain proportional to number of integrated samples is present. In the case when input $S N R$ is low $\left(S N R_{I N}<<1\right)$ output SNR can be worse than input one. Thus it is possible to determine the point separating two types of method behavior corresponding to low and high SNR values which is reversely proportional to the number of samples $N$. Concluding, it should be stressed that the advantage of the analyzed method, besides of SNR threshold, is integration gain and cross terms cancellation. However this advantage is taken in the expense of computation complexity.

\section{Examples of simulation}

Theoretical approach has been examined on the base of simulation. Signal parameters have been taken to be close to the real radar signals parameters (particularly with respect to pulse-width and intermediate frequency values). Results of simulation have presented in Fig.15-22. Presented are LFM 
overlapping in frequency and time signals, having the same or opposite time-tofrequency slopes. Such situation is typical in dense radar signal environment. The task is to distinct signals. The problem has been examined experimentally with purpose to determine to detect and separate signals.
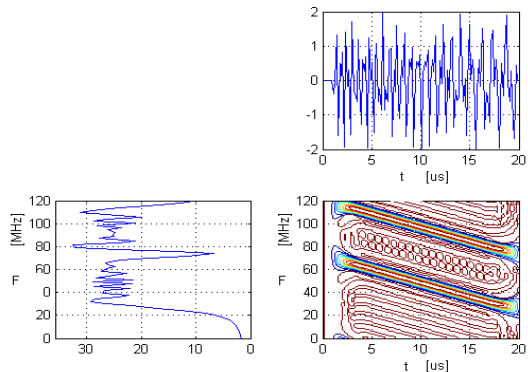

Figure 15: Waveform, spectrum and WV of two overlapping signals.

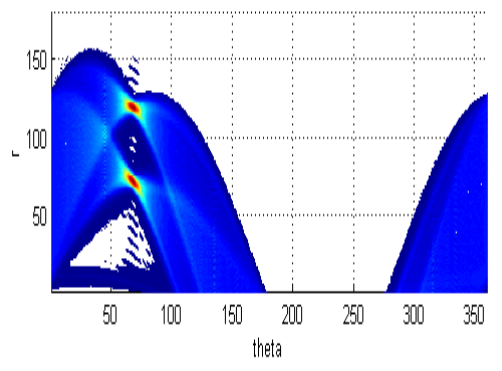

Figure 17: WHT projection onto parameters plane.

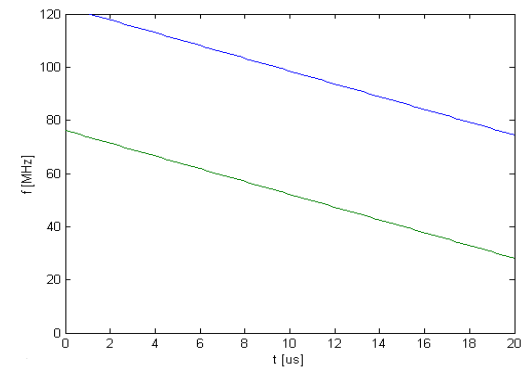

Figure 18: Extracted lines from WHT.
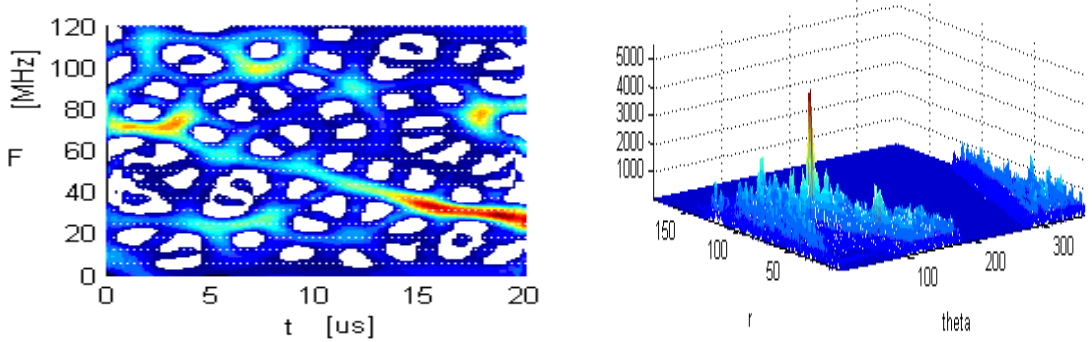

Figure 19: WV of two overlapping signals, $\mathrm{SNR}=-3 \mathrm{~dB}$.
Figure 20: WHT of two overlapping signals, $\mathrm{SNR}=-3 \mathrm{~dB}$.



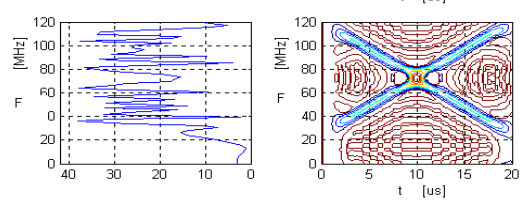

Figure 21: Waveform, spectrum and WV of two overlapping signals.

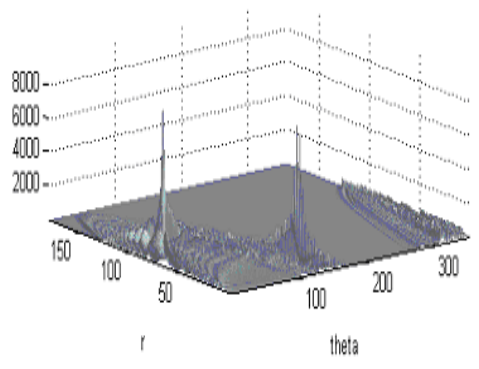

Figure 22: WHT of two overlapping signals.

The results of examinations show that using WHT transform it is possible to extract signal when it is hidden in the noise. Overlapping signals are separated correctly. In the case of simultaneous signals $S N R$ is lowered considerably, as a result of cross terms in $W V$ image. Example presented in Fig. 21 is interesting for the reason, that signals overlap in frequency and time what is not rare in dense radar signal environment.

\section{Conclusions}

The compressive receiver is an efficient tool for the time-frequency signal analysis. Its speed of performance recommends it to apply in radar signal environment to measure frequency parameters, especially of the short-time narrowband and wideband LFM signals. Because the CR output signal represents the instantaneous spectrum of the input signal, the pulse time of arrival is needed. The CR output signals are extremely short (narrow) and their time of appearance at the output has stochastic nature implied by random temporal relations between the reference and input signals. This will cause losses at CR output signal. The main conclusion is that the compressive receiver is high speed Fourier transformer, that aided by task oriented software realizing functions of the virtual measurement instrument provides effective possibility to measure frequency parameters of radar signals. The CR most significant feature is ability to analyze overlapping in frequency and time signals that recommends CR to use in dense signal environment, typical for ELINT/ESM applications. The combination of time-frequency distribution, with a technique of image processing creates new possibilities in signal processing transforms signal detection and parameters estimation to a problem of lines detection in the image. The image is represented by time-frequency distribution of a signal. This approach can be also applied to non-modulated signals and to signals having nonlinear type of frequency modulation. The combined Wigner-Hough transform is robust against disturbances in the image. The method is also effective when segmentation of low quality images is used. This a considerable advantage of Hough transform, because it is possible to extract particular shapes in distorted 
images. Such a property is successfully used for parameters estimation of low power LFM signals especially those existing in the noise. Basic disadvantage of Hough transform is its requirement for a big memory for accumulator and considerable computation complexity. This however is overcome by the use of parallel processing. Current status of problem analyzed here allow to state that there is a need of practical experiment in which the WHT technique will be applied to process signal existing on the ELINT/ESM receiver output with purpose to detect signal and to measure parameters of intrapulse modulation.

\section{Acknowledgements}

This work was supported by the National Center for Research and Development for the years 2007-2010 under Commissioned Research Project PBZ-MNiSWDBO-04/I/2007.

\section{References}

[1] Breuer K.D., J. S. Levy J.S., Paczkowski H.C., The compressive receiver, A versatile tool for EW systems; Microwave Journal, vol.10, (October 1989), pp. 81-98.

[2] Campbell C.K., Application of surface acoustic wave and shallow bulk acoustic wave devices Proceedings of the IEEE, vol. 77, no.10, (October 1989), pp. 1453-1484.

[3] Kawalec A., Pieniężny A., Radar signal feature extraction system based on a saw dispersive delay lines, Molecular and Quantum Acoustics, vol. 26, Poland 2005, pp. 175-182

[4] Kočemasov V.N., Dolbnja E.V., Sobol' N.V., Akustoelektronnye fur'eprocessory, Radio i Svjaz', Moskva, 1987, in Russian.

[5] Moule G.L., SAW compressive receiver for radar intercept, IEE Proceedings, pt. F, Communications, Radar and Signal Processing, vol. 129, no. 3, (1982), pp. 180-186.

[6] Paradowski L., About some version of SAW chirp spectrum analyzer analysis and performance, Proceedings of the 5th Conference on ACOUSTOELECTRONICS'91, 10-13 September, 1991, Varna, Bulgaria, World Scientific Publishing Co. Pte. Ltd., Singapore, 1991, pp. 123-131.

[7] Paradowski L., Pieniężny A., Radar signal processing in time microscale using frequency-to-time and time-to-frequency procedures and transducers, The Proceedings of The 7th International Conference on Signal Processing Applications \& Technology, Boston, Massachusetts, USA, October 7-10, 1996, Miller Freeman Inc., 1996, vol. 2, pp.1500-1504.

[8] Tsui J.B.Y., Microwave receivers with electronic warfare applications, John Wiley and Sons, New York, 1986, pp. 278-328.

[9] Barbarosa S., Lemoine O., Analysis of nonlinear FM signals by pattern recognition of their time-frequency representation, IEEE Signal Processing Letters, vol. 3, no. 4, April 1996. 
[10] Barbarosa S., Analysis of multicomponent LFM signals by a combined Wigner-Hough transform, IEEE Transactions on Signal Processing, vol. 43, no. 6, June 1995.

[11] Claasen T.A.C.M., Mecklenbrauker W.F.G., The Wigner distribution - A tool for time-frequency signal analysis, Part I: Continuous time signals. Philips Journal of Research, vol.35, no.3, 1980.

[12] Cohen L., Time-frequency distribution - A Review, Proceedings of the IEEE, vol.77, no.7, July 1989.

[13] Schleher D.C., Introduction to electronic warfare, Artech House Inc., Dedham, MA, 1986.

[14] Stephens J.P., Advances in signal processing for Electronic Warfare, IEEE AES Systems Magazine, November 1996.

[15] Żorski W., Metody segmentacji obrazów oparte na transformacie Hougha, Instytut Automatyki i Robotyki Wydział Cybernetyki WAT, Warszawa 2000, in Polish. 